\section{RECENT ADVANCES IN THE THEORY OF THE FUNDAMENTAL PARTICLES OF PHYSICS}

\author{
By Prof. R. H. FOWLER, O.B.E., F.R.S.
}

A $\mathrm{T}$ the meeting during January 2-4 in Calcutta of the Indian Science Congress, Dr. H. J. Bhabha was president of the Section of Physics. His presidential address on the theory of the "Fundamental Particles of Physics", to which he has himself made important contributions, has recently been received in Great Britain.

Dr. Bhabha starts by reviewing the manner in which the basic concepts of physics have been generalized from those of the nineteenth century, first by the incorporation of the unified space-time of the theory of relativity and then of the requirements of the quantum theory. This process of incorporation is still incomplete, but significant progress has been made, about which Bhabha's address contains, as one would expect, much interesting comment.

Bhabha starts by reminding us of the fact-which we all know but sometimes forget-that, when the recognition of new facts of observation requires the use of wider postulates than heretofore as the basic postulates of physics, the generalized theory is inevitably more, not less, exacting in its demands on the fundamental equations of physics. The generalization results in less, not more, freedom of choice of quantities that the theory determines $a$ priori. For example, the special theory of relativity requires the fundamental equations of physics to be invariant for the transformations of the Lorentz group, whereas in Newtonian physics the laws need only be invariant for all transformations of the three-dimensional spacerotation group, which is a sub-group of the Lorentz group. It is important to realize that it is just this generalization which eliminates much arbitrariness from Newtonian physics. In Newtonian theory, the interaction between two particles can be chosen entirely arbitrarily and only determined empirically by observation. In the relativistic generalization, the interaction can no longer be given by an arbitrary static function, for such a specification would not be invariant for the extended Lorentz group of transformations. In relativistic theory the interaction between two particles at a distance must be carried by the medium of a field, and the field functions, $u$, of this field must satisfy the generalized wave equation

$$
\frac{\partial^{2} u}{c \partial t^{2}}-\frac{\partial^{2} u}{\partial x^{2}}-\frac{\partial^{2} u}{\partial y^{2}}-\frac{\partial^{2} u}{\partial z^{2}}+\chi^{2} u=0,
$$

in which $\chi$ is an invariant which may be zero. The assertion "must satisfy" above is strictly an overstatement of the logical position. The $u$-equation given is the simplest possible invariant differential equation which $u$ can satisfy and perform its required functions. There are, however, more complicated equations of higher order which $u$ might satisfy alternatively, but these can still rightly be ruled out on grounds of less simplicity, until by exhaustive study it has been found that the observed facts demand the greater complication. For the present we are not so faced, and may provisionally conclude that relativistic invariance restricts us (in the static case) to a variation of the potential energy of interaction of two particles, a distance $r$ apart, of the form

$$
\frac{e-\chi r}{r} \text {, }
$$

or a combination of this function and its space derivatives.

The requirements of the generalization of classical mechanics to cover the quantum theory add to these relativistic restrictions an entirely distincet set of additional restrictions; the combined effect of relativity plus quantum theory is so stringent that one can formulate precisely all the theories that are possible in conformity with both generalizations, and Bhabha describes in his address the various arguments and stages in this notable development, pointing out where the formulation is still inadequate. An important point established even by an incomplete formulation of relativistic quantum theory is that a one-particle theory is impossible. Even if one starts with one particle, there is always the possibility that the number can be increased by pair creation. Recognizing this, one sets out with the objective of a many-particle theory, in which the number of particles is not conserved. Such a theory requires the use of two tensors at least : (i) an energy-momentum tensor $\partial$, one component of which gives the energy density of the particles at any point of spacetime; and (ii) a charge-current tensor, one component of which gives the charge density at any point of space-time. Such a theory can be formulated, but for particles of half odd-integral spin the total energy is not necessarily positive. This is the generalized form of the difficulty first met with by Dirac in his relativistic theory of the electron. It can be satisfactorily turned in the same way as in Dirac's theory, if the particles obey the Fermi-Dirac statistics, and a vacuum corresponds to conditions in which the negative energy states are all full. One concludes that a physically sensible theory of particles of half odd-integral spin $\left(\frac{1}{2} \hbar, \frac{3}{2} \hbar, \frac{5}{2} \hbar, \ldots ..\right)$ is only possible if the particles obey the Fermi-Dirac statistics. Somewhat similar arguments first completed by Pauli and Fierz enable one to conclude that a physically sensible theory of particles of integral spin $(\hbar, 2 \hbar$, $3 \hbar, \ldots .$.$) is only possible if the particles obey the$ Einstein-Bose statistics.

These conclusions as to the relation between spin and statistics of possible fundamental particles are stressed by Bhabha as a remarkable example of how widening the basic concepts of a theory, as here by the unification of relativity and quantum theory, leads to a drastic reduction in the outstanding arbitrariness in the theory and to a consequent advance in our understanding of the basic properties of the fundamental particles of physics.

The address next describes recent progress that has been made in eliminating infinities and divergent results from the relativistic (non-quantum) theory of the point electron. It has been realized recently (the first indication was the discovery of the neutron) that tihe finite inertial mass of a moving charge need not, and in fact can not, be tied on its non-zero size as in Lorentz's theory of the electron. It is best at the present stage to regard mass as a constant of a fundamental particle not determined by the relativistic quantum theory formulation, of the depth discussed in this address, and to abandon any attempt to assign a structure to a fundamental particle. The problem then arises of developing a satisfactory infinity-free theory of a structureless point-particle, reacting with a radiation field. Much progress has 
already been made with the problem. Dirac and Pryce have shown that such a relativistic classical theory of a point electron is possible, taking into account exactly its interaction with the field of electro-magnetic radiation. This success has been extended by Bhabha himself to particles with charge, spin and dipoles, and to such particles moving also in 'meson' fields - that is, fields of the type that arise when the particles interact with each other by continual creation and reabsorption of mesons or heavy electrons of either sign, as appears to be the habit of protons and neutrons. The development of this exact classical theory appears to be a step of great importance, although it has not yet been successfully incorporated in the quantum theory. It is, of course, possible that the extended formulation necessary for this step is so profound that it will turn out to require the fixing of the at present arbitrary inertial masses and dipole strengths attributed to the fundamental particles, which survive in the present exact classical theory-perhaps somewhat after the manner of Eddington's generalized theory. This is a problem for the future : discussion of Eddington's generalizations lies outside the range covered by Bhabha's address.

The programme for the formulation of a relativistic quantum theory contemplated in Bhabha's address is, as we have seen, as yet incompleted and perhaps incompletable. None the less, it is of great interest to panse a moment and consider what sort of world of fundamental particles it could provide us with, and how they would satisfy the requirements of observation. We shall give full weight to all considerations of maximum allowable simplicity, such as assuming that the fundamental particles only have charges $\pm e$ or zero, and no electric dipole moment in a co-ordinate system in which they are at rest, and that particles of unobserved type do not exist unless, of course, their existence follows theoretically from the existence of particles of a type required by observation. On this basis the completed programme would provide us with a set of light 'point' particleselectrons, positrons and neutrinos with charges $-e$, te and $\theta$ respectively, all with spin $\frac{1}{2} \hbar$ and obeying the Fermi-Dirac statistics. The electron and positrons have identical masses and magnetic dipole moments, but the actual values are not fixed by the programme, nor are the mass and dipole moment of the neutrino, which mav differ from those of the electron. The mass of the neutrino is required by observation to be very small compared with that of the electron, and may well be zero. The light particles interact with each other through their electromagnetic fields.

The programme provides us also with a set of heavy 'point' particles-protons (negative protons) and neutrinos of charges $e,-e$ and $0, \operatorname{spin} \frac{1}{2} \hbar$ and obeying the Fermi-Dirac statistics. The protons and negative protons have identical masses and magnetic dipole moments, but the actual values are not fixed by the programme, nor are the mass and dipole moment of the neutron, which may (and in fact do) differ from those of the proton. One would not expect to have observed negative protons. The heavy particles interact with each other through both electromagnetic and meson fields.

The programme also provides us with a set or sets of intermediate particles or mesons, the creation and annihilation of which provide the essential meson field mentioned above. Mesons of charge $\pm e$ and a mass about 170 times that of the electron will provide a meson field of essentially the correct characteristics. Such particles can be unstable and capable of spontaneous conversion into a light electron and neutrino. Such instability is required to account for natural B-ray radioactive decay and for the properties of free mesons as observed in the cosmic rays. Further details are as yet scarcely clear.

The fundamental particles of the programme are thus a rather numerous family of a structure not so simple as the purist might desire before attaching to his satisfaction the label fundamental. None the less, such a synthesis or enumeration when completed would represent a magnificent advance, not only for the parts of the theory that it tidies up, but also for the untidy ends lying about which it emphasizesthe undetermined inertial masses and magnetic dipole moments with their rather surprisingly queer ratios. It is here that this programme, which one might describe as the classical relativistic-quantal programme, appears to have reached the bounds of its foreseeable development. One may reasonably expect that further progress will require profound reformulation-proceeding possibly, as suggested above, along paths already indicated by Eddington.

\section{NATIONAL FLOUR AND BREAD THIRD REPORT}

From the Scientific Adviser's Division, Ministry of Food

7 HE first and second reportsi,2 covered the analyses of 622 samples of flour and 459 samples of commercial bread received up to early October 1942. The present report deals with a further 379 samples of flour and 381 samples of bread received in the period ending May 8, 1943.

\section{Quality of Flour}

In the period under review, certain changes have been made in the composition of National flour. There has been an increase in the percentage of home-grown wheat used, which at present averages 4:3.6 per cent. Since mid-March the addition of $2 \mathrm{lb}$. of skim milk powder per sack has been general $(90-95$ per cent of the flour is now so fortified) while from February 13, barley or a mixture of groats (dehulled oats) and barley has formed part of the grist.

In May 1942, a start was made on the addition of calcium carbonate (in the form of creta præparata) to flour at the rate of $7 \mathrm{oz}$. per sack, and at the end of April 1943, approximately 89 per cent of the National flour produced in the United Kingdom was so fortified.

Fibre and $B_{1}$. The following table shows the different levels of fibre and $B_{1}$ over the 379 samples of flour.

\begin{tabular}{|c|c|c|c|}
\hline \multicolumn{2}{|c|}{ Fibre } & \multicolumn{2}{|c|}{$B_{1}$} \\
\hline $\begin{array}{c}\text { Value } \\
\text { per cent }\end{array}$ & $\begin{array}{l}\text { Per cent of } \\
\text { Samples }\end{array}$ & I.U./gm. & $\begin{array}{l}\text { Per cent of } \\
\text { Samples }\end{array}$ \\
\hline $\begin{array}{c}<0.4 \\
0.5 \\
0.55 \\
0.6 \\
0.7 \\
0.8 \\
0.9\end{array}$ & $\begin{array}{r}9 \cdot 8 \\
33 \cdot 8 \\
49 \cdot 6 \\
61 \cdot 5 \\
84.5 \\
93 \cdot 1 \\
97 \cdot 8\end{array}$ & $\begin{array}{c}>1 \cdot 20 \\
1 \cdot 10 \\
1 \cdot 05 \\
1 \cdot 00 \\
0 \cdot 90 \\
0 \cdot 80\end{array}$ & $\begin{array}{r}4 \cdot 5 \\
23 \cdot 0 \\
40 \cdot 1 \\
67 \cdot 8 \\
92 \cdot 4 \\
98 \cdot 0\end{array}$ \\
\hline
\end{tabular}

A correction has been made in the fibre figures to allow for the addition of any Canadian G.R. flour.

The 'national average' figures for fibre and $B_{1}$ are 